\title{
WIENER TAUBERIAN THEOREM FOR RANK ONE SYMMETRIC SPACES
}

\author{
RUdra P. SARKAR
}

In this article we prove a Wiener Tauberian (W-T) theorem for $L^{p}(G / K), p \in[1,2)$, where $G$ is one of the semisimple Lie groups of real rank one, $S U(n, 1), S O(n, 1), S p(n, 1)$ or the connected Lie group of real type $F_{4}$, and $K$ is its maximal compact subgroup. W-T theorem for noncompact symmetric space has been proved so far for $L^{1}\left(S L_{2}(\mathrm{R}) / S O_{2}(\mathrm{R})\right)$ where the generator is necessarily $K$-finite $([\mathrm{S}])$. We generalize that result to the case of $L^{p}$ functions of real rank one groups, without any $K$-finiteness restriction on the generator. We also obtain a reformulation of the W-T theorems using Hardy's theorem for semisimple Lie groups.

\section{Introduction.}

The purpose of this article is to prove a Wiener Tauberian (W-T) theorem for the Riemannian symmetric space $G / K$ of non compact type, where $G$ is one of the following semisimple Lie groups of real rank one, namely $S U(n, 1)$, $S O(n, 1), S P(n, 1)$ and the connected Lie group of real type $F_{4}$, and $K$ is a maximal compact subgroup of $G$. Most of the notation used in the introduction is standard. The rest will be explained in the next section.

W-T theorems for symmetric space have been proved so far only for the case $\mathrm{SL}_{2}(\mathbb{R}) / \mathrm{SO}_{2}(\mathbb{R})$ by Sitaram $[\mathbf{S}]$ and Sarkar $[\mathbf{S a}]$; Sitaram $[\mathbf{S}]$ extends the W-T Theorem for biinvariant functions in $L^{1}\left(S L_{2}(\mathbb{R})\right.$ ) (proved in Ehrenpreis-Mautner $[\mathbf{E}-\mathbf{M}])$ to a W-T theorem for $L^{1}\left(S L_{2}(\mathbb{R}) / S O_{2}(\mathbb{R})\right)$ where the generator is necessarily a $K$-finite function. A paricular case $(n=0)$ of Theorem 1.1 of [Sa] removes this restriction of $K$-finiteness condition on the generator and extends it for $p \in[1,2)$. In this article we provide an exact analogue of the latter result for $G / K$ as above. We show that if the Fourier transforms of a set of functions in $L^{p}(G / K)$ do not vanish simultaneously on any $L^{p-\varepsilon}$-tempered representations (for some $\varepsilon>0$ ), relevant for functions of $G / K$, and if one of these functions has a Fourier transform which is 'not-too-rapidly-decreasing at $\infty$ ' in a certain sense, then this set of functions generate $L^{p}(G / K)$ as a left $L^{1}(G)$ module.

In switching over from $S L_{2}(\mathbb{R})$ to other groups of real rank 1 , one encounters a number of difficulties which prevent a straight forward extension of 
W-T theorem from $S L_{2}(\mathbb{R}) / S O_{2}(\mathbb{R})$ to other rank 1 symmetric space. To reduce the problem to the biinvariant case (of $S L_{2}(\mathbb{R})$ ) $[\mathbf{S}]$ and $[\mathbf{S a}$ ] have a common way: To find a function $g$ such that the left convolute $g * f$ of the generator $f$ is biinvariant and this convolute has nonvanishing Fourier transform wherever $f$ has the same.

But unlike those of $S L_{2}(\mathbb{R})$, non zero $K$-types are not in general one dimensional and hence can accommodate more than one $M$-type. Note that for a function on $G / K$ with a nontrivial $K$-type in the left, the Fourier transform is a matrix valued function. Hence it is posible that two functions $f$ and $g$ of matching $K$ types (i.e., the right type of $f$ is the same as left type of $g$ ) have non zero Fourier transforms at a certain representation; yet $f * g$ has zero Fourier transform at the representation.

As mentioned in Trombi $[\mathbf{T}]$, there are linear dependecies among matrix coefficients of the principal series representations and their derivatives w.r. to $\lambda \in \mathfrak{a}_{\mathbb{C}}^{*}$ at those $\lambda$ at which the asymptotic expansions of those matrix coefficients have singularity. The maximum order of the derivatives involved is one less than the order of sigularity. For $S L_{2}(\mathbb{R})$ the corresponding order of singularity is at most one and hence derivatives do not come into the picture. Therefore various matrix coefficients of the Fourier transform of a $K$-finite function at such a representation are not quite independent of each other. And contrary to what we have experienced in $S L_{2}(\mathbb{R})$, those relations are not "reformulation of embedding of discrete series in principal series" (see $[\mathbf{T}]$ ).

Singularities of the asymptotic expansions of the matrix coefficients of the principal series representations are the points of trouble. Suppose at a generic point $\lambda_{0}$ in the strip $\mathcal{S}^{\gamma}$, a function $f$ on $G / K$ has only one component say $f_{m}$ of left $K$-type $m$ with nonzero Fourier transform $\widehat{f}_{m}$. The proof requires a function $g$ of type $0, m$, so that $\widehat{g}\left(\lambda_{0}\right) \neq 0$. But if $\lambda_{0}$ is one of those points of trouble, then it is possible that though there is $m$ so that $\Phi_{\lambda_{0}}^{m, 0} \neq 0$ it may happen that $\Phi_{\lambda_{0}}^{0, m}(x) \equiv 0$ for all such $m$. This removes all hope of getting a $g$ as required.

All these pose difficulty in tailoring a $g$ which will reduce the generator to a biinvariant function as in the case of $S L_{2}(\mathbb{R})$. We have saved the situation to a large extent by using results of Johnson and Wallach $[\mathbf{J}-\mathbf{W}]$ and Johnson $[\mathbf{J}]$. We may remark here that a stronger version of this is true for $S L_{2}(R)$ and is implicitly instrumental in the success of $\mathrm{W}-\mathrm{T}$ theorem for the whole of $S L_{2}(\mathbb{R})$ in $[\mathbf{S a}]$ (see Remark in Section 3).

Also we propose a change in the basic step: instead of making a single left convolute of the generator to shoulder the responsibility of having nonvanishing Fourier transform at all points $\lambda$, we get, for each point $\lambda$ of the strip, separate left convolute $g_{\lambda} * f$ of the generator $f$ such that Fourier transform of $g_{\lambda} * f$ is non zero (perhaps only) at the point $\lambda$. This sharing 
of responsibilities over the $g_{\lambda}$ 's eases the process of finding them and helps us to overcome some of the obstacles encountered. Besides it avoids the lengthy arguments and constructions used in [Sa].

A disadvantage of using the Corona theorem in this context (used in $[\mathbf{S}]$ for similar extension of $\mathrm{W}-\mathrm{T}$ theorem from biinvariant functions to $\left.L^{1}\left(S L_{2}(\mathbb{R}) / S O_{2}(\mathbb{R})\right)\right)$ is that it can handle only finitely many functions and therefore can not be adapted in the above scheme of using separate $g_{\lambda}$ for every $\lambda$. Here, on the other hand, we have used the full force (overlooked also in $[\mathbf{S a}]$ ) of the $\mathrm{W}-\mathrm{T}$ theorem for biinvariant functions in $[\mathbf{B}-\mathbf{W}]$ where the generator set is infinite.

The subject matter of the last sections is a reformulation of $\mathrm{W}$-T theorem using 'mathematical uncertainty principle' (see $[\mathbf{H}-\mathbf{J}]$ ) in which we make an application of Hardy's Theorem for semisimple Lie groups in the W-T theorems. This transfers the not-too-rapidly-decreasing condition on the Fourier transform of the generator to a decay condition on the generator.

The author is extremely grateful to S.C. Bagchi for countless discussions with him and his many valuable suggestions.

\section{Notation and Preliminaries.}

Unless mentioned otherwise, $G$ will denote one of those connected semisimple Lie groups of real rank 1 , listed in the introduction. Let $K$ be a fixed maximal compact subgroup of $G$ and $\theta$ be the corresponding Cartan involution. Let $\mathfrak{g}, \mathfrak{k}$ be the Lie algebras of $G$ and $K$ respectively and $\mathfrak{k}+\mathfrak{p}=\mathfrak{g}$ be the Cartan decomposition w.r.to $\theta$. Let $\mathfrak{a}$ be a fixed maximal abelian subspace of $\mathfrak{p}$ and let $A=\exp (\mathfrak{a})$. Then $\operatorname{dim}(\mathfrak{a})=1$. Consider the root space decomposition of $\mathfrak{g}$ w.r.to $\mathfrak{a}$. Due to the one dimensionality of $\mathfrak{a}^{*}$ all roots will give rise to the same reflection. In fact, the only possible roots in this case are $\pm \frac{1}{2} \lambda, \pm \lambda, \pm 2 \lambda$ (see [G-V, p. 62]) of which only one, say $\alpha$ is simple and the Weyl group $\mathcal{W}(A) \cong \mathbb{Z}_{2}$. Let $G=K A N$ be the corresponding Iwasawa decomposition. We denote by $M$ (resp. $\widetilde{M}$ ) the centralizer (resp. normalizer) of $A$ in $K$. Then $\mathcal{W}(A) \cong \widetilde{M} / M$. $\mathcal{P}(A)$ stands for the set of parabolic subgroups of $G$ with split part $A$. Conjugation by elements of $\widetilde{M}$ on $N$ induces a transitive group action on $\mathcal{P}(A)$. Now since $M$ normalizes $N$, the Weyl group $\mathcal{W}(A)$ acts (transitively) on $\mathcal{P}(A)$ : Let $\omega$ be the only non-trivial element of $\mathcal{W}(A)$, which takes the positive roots to the negative roots and let $x_{w} \in \widetilde{M}$ be such that and $\pi\left(x_{w}\right) \equiv \omega \in \mathcal{W}(A), \pi$ being the quotient map: $\pi: \widetilde{M} \longrightarrow \widetilde{M} / M$. Then for $P=M A N \in \mathcal{P}(A)$, $P^{\omega}=P A N^{\omega}=P A \bar{N}=\bar{P}$ where $\bar{N}=\theta(N)=x_{\omega} N x_{\omega}^{-1}$. Thus $\mathcal{P}(A)$ consists of two minimal parabolic subgroups $P$ and $\bar{P}$. Also recall that the only nonminimal parabolic subgroup in our case is $G$ itself.

The representations $\pi(P, \sigma, \lambda)$ and $\pi(\bar{P}, \sigma, \lambda)$ are the principal series representations induced from $P$ and $\bar{P}$ respectively where $\sigma \in \widehat{M}$ and $\lambda \in \mathfrak{a}_{\mathbb{C}}^{*}$. 
The Fourier transform of any right $K$-invariant function $f \in L^{p}(G)$ w.r.to the nonspherical principal series is zero. Therefore only the spherical principal series representations $\pi\left(P, \sigma_{0}, \lambda\right)$ ( $\sigma_{0}$ being the trivial representation of $\widehat{M})$ are relevant here. We will denote the spherical representation $\pi\left(P, \sigma_{0}, \lambda\right)$ simply by $\pi_{\lambda}$. They are $L^{p}$ tempered when $\lambda \in \mathcal{S}^{\gamma}=\{\lambda \in \mathbb{C}|| \Re \lambda \mid \leq \gamma \rho\}$, where $\gamma=\frac{2}{p}-1$ and $\rho$ is the half sum of positive roots (see [T, Sec. 4, Definition 1]). The strip $\mathcal{S}^{\gamma}$ augmented by $\varepsilon$ will be denoted by $\mathcal{S}_{\varepsilon}^{\gamma}=\mathcal{S}^{\gamma+\varepsilon}$.

The spherical principal series representations $\pi_{\lambda}$ for each $\lambda \in \mathfrak{a}_{\mathbb{C}}^{*}$ are realised on the same subspace, say $H_{\sigma_{0}}$ of the Hilbert space $L_{2}(K)$ (compact picture). Let us fix an o.n.b. $\left\{e_{\alpha}\right\}$ of $H_{\sigma_{0}}$ of $K$-finite vectors among which $e_{0}$ is the $K$-fixed vector. By matrix coefficients of the representation $\pi_{\lambda}$ we will always mean matrix coefficients w.r. to this $\left\{e_{\alpha}\right\}$. The $\left(e_{r}, e_{s}\right)$-th matrix coefficient of $\pi_{\lambda}$ will be denoted by $\Phi_{\lambda}^{r, s}$. For $f \in L^{p}(G / K)$ and for $\lambda$ in the interior of the corresponding strip $\mathcal{S}^{\gamma}$, the matrix coefficients of the Fourier transforms, $\left(\widehat{f}\left(\pi_{\lambda}\right)\right)_{e_{i}, e_{j}}=\int\left\langle\pi_{\lambda}\left(x^{-1}\right) e_{i}, e_{j}\right\rangle f(x) d x$ exist (it is a consequence of an inequality of Harish-Chandra [T, Section 4, Proposition 3] and of [T, Section 6, (ii), (v)] that $\left\langle\pi_{\lambda}\left(x^{-1}\right) e_{i}, e_{j}\right\rangle$ is an $L^{q}$-function for $\lambda \in \stackrel{\circ}{\mathcal{S}}^{\gamma}$ and $\left.\frac{1}{p}+\frac{1}{q}=1\right)$ and constitute the formal matrix Fourier transform $\widehat{f}\left(\pi_{\lambda}\right)$. If either $\widehat{f}\left(\pi_{\lambda}\right)$ or $\widehat{g}\left(\pi_{\lambda}\right)$ has finitely many nonzero entries and $f * g$ is defined, then $(\widehat{f * g})\left(\pi_{\lambda}\right)$ is given by the matrix multiplication $(\widehat{f * g})\left(\pi_{\lambda}\right)=$ $\widehat{f}\left(\pi_{\lambda}\right) \widehat{g}\left(\pi_{\lambda}\right)$. For the definition of Schwartz spaces $C^{p}(G)$ 's, $p \in[1,2)$ and the characterization of their image under Fourier transform we refer to $[\mathbf{T}$, Sec. 6 and Sec. 8].

For $\delta \in \widehat{K}$ let $\alpha(\delta)=d(\delta) \overline{\chi_{\delta}}$ where $d(\delta)=$ degree of $\delta$ and $\chi_{\delta}=$ character of $\delta$. Let $d k$ be the normalized Haar measure of $K$. Define $\alpha(\delta) * f$ by $(\alpha(\delta) * f)(x)=\int_{K} \alpha(\delta)(k) f(k x) d k$ for all $x \in G$. $f$ is said to be of left type $\delta$ when $\alpha(\delta) * f \equiv f$. A function is of type $(\delta, 0)$ when it is right invariant and its left type is $\delta$. We have extended the notation used in $[\mathbf{B a}]$ and $[\mathbf{S a}]$ to the context of real rank 1 groups where the meaning is unambiguous.

Let us now add some more notation, essentially from $[\mathbf{T}]$. We will denote by $U_{p}$ the set of points of trouble $U$ in the $p$-strip $\mathcal{S}^{\gamma}$. A detailed description of $U$ is available in [T, pp. 103-108]. In brief: Let $U$ be the set of points in $\mathfrak{a}_{\mathbb{C}}^{*}=\mathbb{C}$, at which there are singularities of the asymptotic expansion of the matrix coefficients. Let $F=\{\delta, 0\}$, a set of two $K$-types of which ' 0 ' is the trivial one. Let $e_{1}, e_{2}, e_{3}, \ldots, e_{l}$ are vectors in $\mathcal{H}_{\sigma_{0}}$ which transforms according to $\delta$, where $\sigma_{0}$ is the trivial element of $\widehat{M}$ and $e_{0}$ as ususal is the $K$-fixed vector. Fix a minimal parabolic $P$. Consider the principal series representation $\pi\left(P, \sigma_{0}, \lambda\right)$ for $\lambda \in \mathbb{C}$. Let $\mathcal{E}$ be the set of matrix coefficients of these principal series representations w.r.to vectors $\left(e_{i}, e_{0}\right), i=1,2, \ldots, l$ and their derivatives w.r.to. $\lambda$ at the points $w \lambda$ where $w \in \mathcal{W}$ and $\lambda \in U$. As mentioned earlier, there are linear relations among the elements of $\mathcal{E}$. One 
can choose a basis of $\mathcal{E}$, so that any element of $\mathcal{E}$ is a linear combination of the basis elements.

\section{W-T Theorem for rank one Symmetric spaces.}

Let us first consider the $\mathrm{W}-\mathrm{T}$ theorem for biinvariant functions:

Theorem 3.1. Let $\left\{f^{\alpha} \mid \alpha \in \Lambda\right\}$ be a family of functions in $L^{p}(K \backslash G / K), \Lambda$ being an index set, such that the Fourier transforms $\widehat{f}^{\alpha}$ extend holomorphically to the strip $\mathcal{S}_{\varepsilon}^{\gamma}$ for some $\varepsilon>0$ and all the matrix coefficients of $\widehat{f}^{\alpha}, \alpha \in \Lambda$ vanish at infinity, that is, $\lim _{|\lambda| \longrightarrow \infty}\left|\left(\widehat{f}^{\alpha}(\lambda)\right)_{m, n}\right|=0$ on $\mathcal{S}_{\varepsilon}^{\gamma}$. Suppose that the collection $\left\{\hat{f}^{\alpha} \mid \alpha \in \Lambda\right\}$ does not vanish simultaneously on any point of the $\mathcal{S}_{\varepsilon}^{\gamma}$. Moreover let there be an $\alpha_{0} \in \Lambda$ such that $\widehat{f}^{\alpha_{0}}$ further satisfies the not-too-rapidly-decreasing condition at infinity: $\lim \sup _{|t| \longrightarrow \infty}\left|\left(\widehat{f}^{\alpha_{0}}\right)(i t)\right|\left|e^{K e^{|t|}}\right|>0$ for all $K>0$. Then the $L^{1}(K \backslash G / K)$ module generated by $\left\{f^{\alpha} \mid \alpha \in \Lambda\right\}$ is dense in $L^{p}(K \backslash G / K)$.

We omit the proof of this theorem as it runs entirely along the lines of the corresponding proof for $L^{1}\left(S L_{2}(\mathbb{R})\right)_{0,0}$ in $[\mathbf{B}-\mathbf{W}]$. The crux of the matter is that the space of Fourier transforms of the Schwartz spaces $C^{p}(\widehat{G})_{0,0}$ as a function space is indistinguishable from the corresponding space for $S L_{2}(\mathbb{R})$. And the only difference between $C^{1}(\widehat{G})$ and $C^{p}(\widehat{G})$ is the width of the strip $\mathcal{S}^{\gamma}$, which is the domain of the Fourier transforms.

On our way to the main theorem we need the following:

Observation. For every $\lambda \in \mathbb{C}$, the $K$-fixed vector $e_{0}$ is cyclic in at least one of the spherical principal series among $\left\{\pi_{w \lambda} \mid w \in \mathcal{W}\right\}$. (See Johnson and Wallach $[\mathbf{J}-\mathbf{W}]$, Theorem $5.1(2),(3),(4)$ and Johnson $[\mathbf{J}]$, Theorem 5.2.)

For a fixed $p$ let us fix a $p^{\prime}<p$. Then it is known that $C_{\mathbb{C}}^{\infty} \subset C^{p^{\prime}} \subset C^{p}$. When $p>1$ we will take $p^{\prime}=1$. Then we have:

Lemma 3.2. Let $\lambda \in \mathcal{S}_{\varepsilon}^{\gamma}$ and let $\pi_{w \lambda}$ be the spherical principal series representation in which $e_{0}$ is a cyclic vector. Suppose for some $f \in L^{p}(G / K)$, $\widehat{f}\left(\pi_{w \lambda}\right) \neq 0$. Then there is a $g \in C^{1}(G) \cap C^{p^{\prime}}(G)$ such that $g * f$ is a biinvariant $L^{p}$-function and $\widehat{g * f}\left(\pi_{w \lambda}\right) \neq 0$.

Proof. For any $K$-type $\delta$, let ${ }_{\delta} f$ be the projection of $f$ in the left $K$-type $\delta$. Then $\delta f$ is a $(\delta, 0)$ type function. Its Fourier transform will be a column vector. $\widehat{f}\left(\pi_{w \lambda}\right) \neq 0$ implies that there is a $K$ type $\delta$ so that $\delta \widehat{f}$ is nonzero at $\pi_{w \lambda}$. This means that there is a vector $e_{r}$ in $\pi_{w \lambda}$ which transforms according to $\delta$, and $\left(e_{r}, e_{0}\right)$-th matrix coefficient of the Fourier transform of $f$ at $\pi_{w \lambda}$ is nonzero. Now as the $K$-fixed vector $e_{0}$ is cyclic in $\pi_{w \lambda}$, the matrix coefficient $\left\langle\pi_{w \lambda}(x) e_{0}, e_{r}\right\rangle$ can not be indentically zero, since otherwise the closed linear span of $\left\{\pi_{w \lambda}(x) e_{0} \mid x \in G\right\}$ will be a subrepresentation orthogonal to $e_{r}$, 
contradicting the fact that $e_{0}$ is cyclic in $\pi_{w \lambda}$. If $\lambda \in \mathcal{S}^{\gamma}-\left\{w \cdot U_{p} \mid w \in \mathcal{W}\right\}$ then it is clear from the description of $C^{1}(\widehat{G})$ and its isomorphism with $C^{1}(G)$ ([T, Sec. 8, Definition 1] and [T, Sec. 11, Theorem 1]), that there exists $g \in C^{1}(G)$ of the type $(0, \delta)$ such that only the $\left(e_{o}, e_{r}\right)$-th matrix coefficients of its Fourier transform is nonzero at $\pi_{w \lambda}$. It readily follows that $g * f$ is a biinvariant function with $\widehat{g * f}\left(\pi_{w \lambda}\right) \neq 0$.

Now when $\lambda \in \mathcal{S}^{\gamma} \cap\left\{w \cdot U_{p} \mid w \in \mathcal{W}\right\}$, it is not clear how to find a $g$ as above which will have only one chosen matrix coefficient nonzero as this time the matrix coefficients have dependencies among themselves $[\mathbf{T}$, Sec. 8, Definition 1(4)]. We need a more careful argument here to show that such a $g$ is available. Since $e_{0}$ is cyclic for $\pi_{w \lambda}$, its $\left(e_{0}, e_{r}\right)$-th matrix coefficient, $\Phi_{w \lambda}^{0, r}$ can not be identically zero. Hence this particular matrix coefficient can be in the basis mentioned in Section 2. Also for the same reason, for linearly independent vectors $e_{r_{1}}, e_{r_{2}} \ldots, e_{r_{k}} \in \mathcal{H}_{\sigma_{0}}$ it can not happen that $\Sigma_{i=1}^{k} a_{i}\left\langle\pi_{w \lambda}(x) e_{0}, e_{r_{i}}\right\rangle=\left\langle\pi_{w \lambda}(x) e_{0}, \Sigma_{i=1}^{k} a_{i} e_{r_{i}}\right\rangle=0$ for all $x \in G$, unless $a_{1}=$ $a_{2}=\ldots=a_{k}=0$. Thus the matrix coefficients $\Phi_{w \lambda}^{0, r}$ are linearly independent functions for $r=1, \ldots, k$, where $e_{1}, \ldots, e_{k}$ and $e_{0}$ form a basis of the space of vectors transforming according to $\delta$ in the representation space $\pi_{w \lambda}$. However they may depend on some of the derivatives of the others. Now as $\Phi_{w \lambda}^{0, r}$ are linearly independent elements of $\left(C^{1}(G)_{0, \delta}\right)^{*}$, the dual space of the Frechét space $C^{1}(G)_{0, \delta}$, an application of Hahn-Banach thorem now gives us a $g \in C^{1}(G)_{0, \delta}$ such that only its $\left(e_{0}, e_{r}\right)$-th matrix coefficient of the Fourier transform is not zero. One can also appeal directly to isomorphism of $C^{1}(G)$ with $C^{1}(\widehat{G})[\mathbf{T}$, Sec. 11 , Theorem 1] to get such a $g$. This proves the lemma for $p>1$ as in this case $p^{\prime}$ can be taken to be 1 .

When $p=1$ we proceed through the same steps. Only instead of appealing to the isomorphism theorem of schwartz spaces $C^{p^{\prime}}$, we use Paley-Wiener Theorem (see Kawazoe [Ka, Theorem 5.2]) for getting a $g$ as above. As $C_{\mathbb{C}}^{\infty} \subset C^{p^{\prime}} \subset C^{1}$ for any $p^{\prime}<1$, the lemma follows.

Note that in the above proof the choice of the function $g$ depends on $\lambda$. It serves only for $\lambda$.

Theorem 3.3. Let $\left\{f^{\alpha} \mid \alpha \in \Lambda\right\}$ be a subset of $L^{p}(G / K), \Lambda$ being an index set, such that the Fourier transform $\widehat{f}^{\alpha}$ of each $f^{a}$ has a holomorphic extension on $\stackrel{\circ}{\mathcal{S}}_{\varepsilon}^{\gamma}$ for some $\varepsilon>0$ and all the matrix coefficients of $\widehat{f}^{\alpha}$ for all $\alpha$ vanish at infinity, i.e., $\lim _{|\lambda| \longrightarrow \infty}\left|\left(\hat{f}^{\alpha}(\lambda)\right)_{m, n}\right|=0$ on $\mathcal{S}_{\varepsilon}^{\gamma}$. Suppose that the collection $\left\{\widehat{f}^{\alpha} \mid \alpha \in \Lambda\right\}$ do not have common zero on any representation (containing the $K$-fixed vector) parametrized by $\mathcal{S}_{\varepsilon}^{\gamma}$. Let there be an $\alpha_{0} \in \Lambda$ such that $\widehat{f}^{\alpha_{0}}$ further satisfies the condition:

$$
\limsup _{|t| \longrightarrow \infty}\left\|{ }_{\delta}\left(\widehat{f}^{\alpha_{0}}\right)(i t)\right\|\left|e^{K e^{|t|}}\right|>0 \quad \text { for all } K>0,
$$


for some $\delta \in \widehat{K}$. Then the left $L^{1}(G)$ module generated by $\left\{f^{\alpha} \mid \alpha \in \Lambda\right\}$ is dense in $L^{p}(G / K)$.

Proof. We look at the collection of $K$-biinvariant functions:

$$
\mathcal{F}=\left\{g * f^{\alpha} \mid g \in C^{1}(G) \cap C^{p^{\prime}}, \text { and } g \text { is left invariant, } \alpha \in \Lambda\right\},
$$

where $p^{\prime}$ is as in Lemma 3.2. Without loss of generality we assume that the strip $\mathcal{S}_{\varepsilon}^{\gamma}=\mathcal{S}^{\gamma+\varepsilon}$ corresponds to $p^{\prime}$, i.e. $\frac{2}{p^{\prime}}-1=\gamma+\varepsilon$; otherwise $\gamma+\varepsilon$ can be replaced by $\min \left\{\gamma+\varepsilon, \frac{2}{p^{\prime}}-1\right\}$. Let $\lambda \in \mathcal{S}_{\varepsilon}^{\gamma}$. Then there exists $w_{0} \in \mathcal{W}$ such that the $K$-fixed vector $e_{0}$ is cyclic in $\pi_{w_{0} \lambda}$. By the hypothesis there is $\alpha \in \Lambda$ such that $\widehat{f}^{\alpha}\left(w_{0} \lambda\right) \neq 0$. Therefore by the lemma above there is a member $g * f^{\alpha}$ in $\mathcal{F}$ for which $\widehat{g * f^{\alpha}}\left(w_{0} \lambda\right) \neq 0$. But $g * f^{\alpha}$ being a $K$-biinvariant function $\widehat{g * f^{\alpha}}(\lambda)=\widehat{g * f^{\alpha}}\left(w_{0} \lambda\right)$ and hence $\widehat{g * f^{\alpha}}(\lambda) \neq 0$. Thus the collection $\mathcal{F}$ satisfies the nonvanishing condition of Theorem 3.1.

The not-too-rapidly-decreasing condition in the hypothesis implies,

$$
\limsup _{|t| \longrightarrow \infty} \|{ }_{\delta}\left(\widehat{f}^{\alpha_{0}}\right)(i t)_{n, 0}||\left|e^{K e^{|t|}}\right|>0
$$

for all $K>0$, where $\delta\left(\widehat{f}^{\alpha_{0}}\right)(i t)_{n, 0}$ is the matrix coefficient w.r.to. the vectors $\left(e_{n}, e_{0}\right)$ for some vector $e_{n}$ in $\mathcal{H}_{\sigma_{0}}$ which transforms according to $\delta$. We find a function $g \in C^{1}(G)_{0, \delta}$ which is left invariant and the only nonzero component of its Fourier transform is $\widehat{g}_{0, n}$ and further $\left|\widehat{g}_{0, n}(i t)\right|$ is of order $e^{-t^{2}}$ for $t \in \mathbb{R}$. Such a choice is possible because except for $t=0$, all other representations parametrized by $\lambda=i t, t \in \mathbb{R}$ are irreducible representations (see Knapp $[\mathbf{K}]$, Theorem 14.15). Therefore the matrix coefficients are linearly independent since any linear relation between two matrix coefficients say, $\left\langle\pi(x) e_{0}, u\right\rangle$ and $\left\langle\pi(x) e_{0}, v\right\rangle$ for two linearly independent vectors $u, v$ in the irreducible representation $\pi$ would mean $\left\langle\pi(x) e_{0}, u-k v\right\rangle \equiv 0$. This implies that the closed linear span of $\pi(x) e_{0}$ for $x \in G$ is a subrepresentation orthogonal to $u-k v$, contradicting the irreducibility of $\pi$. Then $g * f^{\alpha_{0}}$ is $K$-biinvariant and $\widehat{g * f^{\alpha}}$ satisfies the decay condition of Theorem 3.1. As $g \in C^{p^{\prime}}$, for $p^{\prime}<p$ all other conditions of Theorem 3.1 are clearly satisfied. Therefore by that theorem the $L^{1}(G)$-module generated by $\mathcal{F}$ is dense in $L^{p}(G)_{0,0}$. Now as the smallest closed left $L^{1}(G)$-invariant subspace of $L^{p}(G / K)$ containing $L^{p}(G)_{0,0}$ is $L^{p}(G / K)$ itself, the theorem follows.

\section{Remarks.}

1. The results of $[\mathbf{J}-\mathbf{W}]$ and $[\mathbf{J}]$ mentioned above plays an important role for the success of this theorem. A more general and stronger result is true for $S L_{2}(\mathbb{R})$ : Fix a $K$ type $n$, which determines a single $M$ type $\sigma$. Then for every $\lambda,\left\{\pi_{w \lambda} \mid w \in \mathcal{W}\right\}$ has at least one element which has an irreducible subrepresentation containing a vector which transforms according to $n$. It 
is a key fact behind the sucess of the $\mathrm{W}$-T theorem for whole of $L^{p}\left(S L_{2}(\mathbb{R})\right)$ in $[\mathbf{S a}]$.

2. The slightly bigger strip is a technical necessity inherited from the corresponding results for the $K$-biinvariant functions $([\mathbf{E}-\mathbf{M}]$ and $[\mathbf{B}-\mathbf{W}])$. The process of extension (from the $K$-biinvariant case) employed in this article, however, do not use the requirement of the bigger strip. This is yet another advantage over $[\mathbf{S}]$, where the Corona theorem is made use of in the extension and their use of the Corona theorem needs an extended domain essentially. Even when one starts from an exact strip version of the W-T theorem for the $K$-biinvariant functions, the use of the Corona theorem adds the restriction of bigger strip, while going towards W-T theorem for symmetric space from that of $K$-biinvariant functions in the way demonstrated in $[\mathbf{S}]$. Recently Ben Natan et al. has provided in [B-B-H-W 2] (announced in $[\mathbf{B}-\mathbf{B}-\mathbf{H}-\mathbf{W}])$ an exact strip version of $\mathrm{W}-\mathrm{T}$ theorem for biinvariant $L^{1}$ functions of $S L_{2}(\mathbb{R})$. For the fact in 1 above, arguments analogus to Theorem 3.3 will lead to an extension of the result $[\mathbf{B}-\mathbf{B}-\mathbf{H}-\mathbf{W}]$ to an exact-strip version of the W-T thorem for $P S L_{2}(\mathbb{R})=S L_{2}(\mathbb{R}) /\{ \pm I\}$ :

Theorem 3.4. Let $\mathcal{F} \subset L^{1}\left(P S L_{2}(\mathbb{R})\right)$. Suppose that the Fourier transforms of the functions in $\mathcal{F}$ never vanish simultaneously on any relevant $L^{1}$-tempered irreducible representations (i.e., on the representations parametrized by the points on the strip $\mathcal{S}^{1}$ and on the discrete series parametrized by odd integers) and that $\delta_{\infty}(\mathcal{F})=0$ where $\delta_{\infty}(\mathcal{F})=\inf \left\{\delta_{\infty}(f)\right\}$ and $\delta_{\infty}(f)=-\lim _{t \longrightarrow \infty} \sup e^{-\pi t} \log |\widehat{f}(i t)|$. Then the ideal generated by $\mathcal{F}$ is dense in $L^{1}\left(P S L_{2}(\mathbb{R})\right)$.

\section{Hardy's theorem and not-too-rapidly-decreasing conditions.}

Here we obtain a reformulation of the W-T theorems by using Hardy's theorem for semisimple Lie groups, proved by Sitaram and Sundari [S-S]. Let us first quote the Hardy's theorems, for noncompact symmetric spaces from [S-S]. Here $\sigma(x)$ is the norm given by the Killing form.

Theorem 4.1 (Sitaram-Sundari). Let $G$ be a connected, noncompact semisimple Lie group $G$ with finite center. Suppose $f$ is a measurable right $K$-invariant function on $G$ satisfying the following estimates for some positive constants $C, \alpha$ and $\beta:|f(x)| \leq C e^{-\alpha \sigma(x)^{2}}, x \in G$ and $\|\widehat{f}(\lambda)\| \leq$ $C e^{-\beta\|\lambda\|^{2}}, \lambda \in i \mathfrak{a}^{*}$. If $\alpha \beta>\frac{1}{4}$, then $f=0$ a.e.

From this we get for $G$ as in the previous sections:

Theorem 4.2. Let $\mathcal{F}=\left\{f^{r} \mid r \in \Lambda\right\}$ be a subset of $L^{p}(G / K), \Lambda$ being an index set, such that the Fourier transforms $\widehat{f}^{r}$ of each $f^{r}$ has a holomorphic extension on $\stackrel{\circ}{\mathcal{S}}_{\varepsilon}^{\gamma}$ for some $\varepsilon>0$ and all the matrix coefficients of $\widehat{f}^{r}$ for all $r$ vanish at infinity, that is, $\lim _{|\lambda| \longrightarrow \infty}\left|\left(\widehat{f}^{r}(\lambda)\right)_{m, n}\right|=0$ on $\mathcal{S}_{\varepsilon}^{\gamma}$. And let for 
some $f \in \mathcal{F}$ and positive constants $C$ and $\alpha,|f(x)| \leq C e^{-\alpha \sigma(x)^{2}}, x \in G$. Also assume that the collection $\left\{\widehat{f}^{r} \mid r \in \Lambda\right\}$ does not have common zeros on $\mathcal{S}_{\varepsilon}^{\gamma}$. Then the left $L^{1}(G)$ module generated by $\left\{f^{r} \mid r \in \Lambda\right\}$ is dense in $L^{p}(G / K)$.

Proof. Take $\beta=\frac{1}{3 \alpha}$. Then by Theorem 4.1 above $\lim \sup \|\widehat{f}(\lambda)\| \cdot e^{\beta|\lambda|^{2}}=\infty$ $|\lambda| \longrightarrow \infty$

for $\lambda \in i \mathbb{R}=i \mathfrak{a}^{*}$. Therefore $\lim \sup _{|\lambda| \longrightarrow \infty}\|\widehat{f}(\lambda)\| \cdot e^{K e^{|\lambda|}}=\infty$ for all $K>0$. Now it is clear that $\mathcal{F}$ satisfies all the conditions of Theorem 3.3. Hence the theorem follows.

\section{Remarks.}

1. Any function $f$ in $C_{c}^{\infty}$ trivially has the decay and so the not-so-rapidlydecreasing condition can be replaced by assuming that the generator set contains a function from $C_{c}^{\infty}$.

2. There is a distinguished space of functions, known as Zero-Schwartz space, on $G$ which contains $C_{c}^{\infty}(G)$ and sits inside $C^{p}(G)$ for every $p \in(0,2]$ (see $[\mathbf{B a}]$ and Wallach $[\mathbf{W}])$. Infact the Zero-Schwartz space, $C^{0}(G)=$ $\cap\left\{C^{p}(G) \mid p \in(0,2]\right\}$. For $f \in C^{0}(G),|f(x)| \leq e^{-K \sigma(x)}$, for all $K>1$ (see $\left[\mathbf{B a}\right.$, p. 99]). One wonders if a function from $C^{0}(G)$ in the generator set can substitute for the not-too-rapidly-decreasing condition.

\section{References}

[Ba] W.H. Barker, $L^{p}$ harmonic analysis on $S L(2, \mathbb{R})$, Memoir of the American Mathematical Society, 393 (1988).

[B-B-H-W] Y. Ben Natan, Y. Benyamini, H. Hedenmalm and Y. Weit, Wiener's Tauberian theorem in $L^{1}(G / / K)$, and harmonic functions in the unit disk, Bulletin AMS, 32(1) (1995), 43-49.

[B-B-H-W 2] W Wiener's Tauberian theorem In $L^{1}(G / / K)$, and harmonic functions in the unit disk, Department of Mathematics, Upsala University Report, 1994.

[B-W] Y. Benyamini and Y. Weit, Harmonic analysis of spherical functions on $S U(1,1)$, Ann. Inst. Fourier, Grenoble, 42(3) (1992), 671-694.

[E-M] L. Ehrenpreis and F.I. Mautner, Some properties of the Fourier transform on semi simple Lie groups I, Annals of Math., 61 (1955), 406-439.

[G-V] R. Gangolli and V.S. Varadarajan, Harmonic analysis of spherical functions on real reductive groups, Ergebnisse der Mathematik und ihrer Grenzgebiete, 101, Springer-Verlag, 1988.

[H-J] V. Havin and B. Joricke, The uncertainty principle in harmonic analysis, Ergebnisse der Mathematik und ihrer Grenzgebiete, 3, Foldge, Bd. 28, Springer-Verlag, 1994.

[J] K.D. Johnson, Composition series and intertwining operators for the spherical pricipal series II, Transactions of the American Mathematical Society, 215 (1976), 269-283. 
[J-W] K.D. Johnson and N.R. Wallach, Composition series and intertwining operators for the spherical pricipal series I, Transactions of the American Mathematical Society, 229 (1977), 137-173.

[Ka] T. Kawazoe, Fourier transform of $L^{p}$ on real rank 1 semisimple Lie groups, J. Math. Soc. of Japan, 34(4) (1982), 561-579.

[K] A.W. Knapp, Representation theory of semisimple groups, Princeton University Press, Princeton, New Jersy, 1986.

[Sa] R.P. Sarkar, Wiener Tauberian theorems for $S L_{2}(\mathbb{R})$, Pacific J. of Math., 177 (1997), 291-304.

[S] A. Sitaram, On an analogue of Wiener Tauberian theorem for symmetric spaces of the non-compact type, Pacific J. of Math., 133 (1988), 197-208.

[S-S] A. Sitaram and M. Sundari, An analogue of Hardy's theorem for very rapidly decreasing functions on semi-simple Lie groups, Pacific J. of Math., 177 (1997), 187-200.

[T] P.C. Trombi, Harmonic analysis of $C^{p}(G: F)(1 \leq p<2)$, Journal of Functional Analysis, 40 (1981), 84-125.

[W] N.R. Wallach, Asymptotic expansion of generalized matrix entries of representations of real reductive groups, Lecture Notes in Math., 1024, SpringerVerlag, (1983), 287-369.

Received August 21, 1996 and revised December 8, 1997. This work is supported by a research award of National Board for Higher Mathematics, India.

Indian Statistical Institute

CALCUTTA 700035

INDIA

E-mail address: rpsarker@hotmail.com 